\title{
Fever in Children - an Assessment of Validity by a Shewhart Model in a Syndromic Surveillance System in China
}

\author{
Changming Zhou*1, Huijian Cheng², Genming Zhao', Qi Zhao', Biao Xu' ${ }^{1}$, Vinod Diwan ${ }^{3}$ \\ and ${\mathrm{Xue} \mathrm{Li}^{4}}^{\mathrm{Z}}$
}

${ }^{1}$ Public Health, Fudan University, Shanghai, China; '2Jiangxi Province Center for Disease Prevention and Control, Nanchang, China; ${ }^{3}$ Karolinska Institutet, Stockholm, Sweden; ${ }^{4}$ Future Position X, Gavle, Sweden

\section{Objective}

To evaluate the validity of a syndromic surveillance system in health facilities of rural China, signals generated by Shewhart charts from the reported febrile patients in children were compared with that from the common infectious disease patients reported to the conventional case report system (CISDCP, China Information System for Disease Control and Prevention)

\section{Introduction}

Since April 2012, an integrated syndromic surveillance system in rural China (ISSC) has been established in health facilities in two rural counties of Jiangxi Province, China [1]. The objective of ISSC is to integrate syndromic surveillance with conventional case report system for the early detection of infectious disease outbreak in rural China.

\section{Methods}

A total of 167 health facilities including 2 county hospitals, 15 township hospitals and 150 village health stations were sampled as surveillance sites in 2 counties in Jiangxi province, China. Health facility visits of patients with ten major symptoms including fever, cough, sore throat, diarrhea, and nausea/vomiting, together with their age and gender were reported to a web-based platform during April 2012 and March 2014. Data on children patients with CID common infectious diseases (CID) in these two counties reported to the CISDCP during the same period were retrieved including measles, hand foot mouth disease (HFMD), chickenpox, rubella, influenza, and mump. The time trend and alert signals in both ISSC and CISDCP were generated by Shewhart model [3] (baseline $=15$ days, lag $=2$ days, $\sigma=3$ ). Sensitivity and PPV were used to compare the signals generated in ISSC with that in CISDCP (using the signals in CISDCP as reference). A matched signal was defined as a signal generated in ISSC having at least one corresponding signal occurred in CISDCP within a duration of 7 days.

\section{Results}

There were 28,049 and 42,029 reports respectively for febrile patients in children from health facilities in these two counties during the two-year period. According to the CISDCP, there were 511 and 1779 selected CID cases reported respectively (Table 1).

The time trend of febrile patients and CID patients with Shewhart signals were illustrated in Figure 1 and figure 2 . The time trend of the two datasets generally matched to each other. However in Jan. 2014 there was a peak in febrile patients in ISSC with no observed changes in CIDs in CISDCP. The sensitivity were $29.03 \%$ and $34.78 \%$. The PPVs were $64.29 \%$ and $53.33 \%$ in the two counties respectively. (Table 2.)

\section{Conclusions}

Conclusion: The sensitivity of signals in the syndromic surveillance is relatively low using the Shewhart model. This might be the result of using the non-specific symptom - fever. HoweverPPV was relatively high in fever in ISSC for detecting CID within children using Shewhart model. These results suggested that this system had potential ability to supplement conventional case report system in detecting common infectious disease outbreaks in children, under the condition that every signal can be verified with high quality by local disease control workers.

Table1. Records of children febrile patients in ISSC and CID in CISDCP

\begin{tabular}{|c|c|c|c|c|c|c|c|}
\hline \multirow{2}{*}{ County } & ISSC & \multicolumn{6}{|c|}{ CISDCP } \\
\cline { 2 - 8 } & Fever & Rubella & Influenza & Mumps & HFMDs & Varicella & Total cases \\
\hline County F & 28049 & 3 & 14 & 37 & 374 & 83 & 511 \\
\hline County Y & 42029 & 2 & 17 & 291 & 1242 & 227 & 1779 \\
\hline
\end{tabular}

Table2. Numbers of signals generated by Shewhart model and sensitivity \& PPV

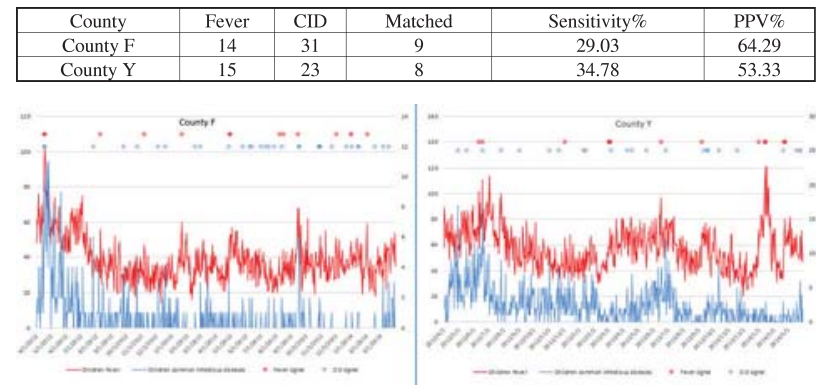

\section{Keywords}

Syndromic surveillance; Shewhart model; children

\section{Acknowledgments}

This study was funded by European Union's Seventh Framework Programmer ([FP7/2007-2013] [FP7/2007-2011]) under grant agreement no. [241900].

We thank all the data collectors and participants in ISSC project in Jiangxi sites.

\section{References}

[1]. Yan, W., et al., ISS - an electronic syndromic surveillance system for infectious disease in rural China. PLoS One, 2013. 8(4): p. e62749.

[2]. The Tenth National People's Congress, Law of the People's Republic of China on Prevention and Treatment of Infectious Diseases. 2004.

[3]. Babcock, G.D., et al., Use of CUSUM and Shewhart charts to monitor regional trends of birth defect reports in New York State. Birth Defects Res A Clin Mol Teratol, 2005. 73(10): p. 669-78.

\section{*Changming Zhou}

E-mail: 12111020001@fudan.edu.cn 